\title{
An Analysis of the Importance of Traditional Zheng Tunes in the Teaching of Zheng in Colleges and Universities
}

\author{
Ying Liu \\ Xiamen University Tan Kah Kee College \\ Zhangzhou, China
}

\begin{abstract}
With the development of society and the improvement of economic level, people are paying more and more attention to the learning of art, and the learning of musical instruments is becoming more and more popular. Zheng is an important musical instrument in ancient China and is loved by more and more people. Therefore, this paper focuses on the importance of traditional Zheng tunes in the teaching of Zheng in colleges and universities, analyzes the problems existing in the current teaching process of traditional Zheng tunes, and proposes corresponding and achievable optimization strategies, hoping to effectively promote the development and progress of this field and the continuous improvement of the effectiveness of Zheng teaching. At the same time, it also provides reference for relevant people who pay attention to this issue, hoping to lead to more thinking and improvement measures.
\end{abstract}

Keywords-traditional Zheng tunes; the teaching of Zheng; importance

\section{INTRODUCTION}

The practice of traditional Zheng tunes plays an important role in the study of Zheng and is an indispensable part of the modern Zheng teaching. Based on this situation, this paper analyzes the importance of traditional Zheng tunes in the teaching process of Zheng in colleges and universities, analyzes the problems existing in the current teaching process of traditional Zheng tunes and proposes corresponding countermeasures, which has important social practical significance and non-negligible value for the development of this field. It is also hoped that through this article, more thinking about this field can be stimulated, the effectiveness of Zheng teaching in colleges and universities can be improved and further research and progress in this field of art can be achieved.

\section{THE IMPORTANCE OF TRADITIONAL ZHENG TUNES IN THE TEACHING OF ZHENG IN COLLEGES AND UNIVERSITIES}

Traditional Zheng tunes are concentrated expressions of art in the process of historical development. The study of traditional Zheng tunes cannot be carried out in isolation. Only by truly understanding the historical development stories and folk customs behind the Zheng tunes can the practitioners master the connotation of the tunes more deeply and improve their understanding and cognition of the tunes. The development of traditional Zheng tunes of different genres is introduced as follows to explain the importance of traditional Zheng tune teaching:

First of all, Henan has been a province in the central plains since ancient times. The development of Zheng tunes in this area also has a long history and is influenced by the culture and art of the central plains. Henan Zheng tunes were greatly influenced by Henan Opera and the folk rap art in Henan in the development process. Henan Zheng tunes have been evolving over the past more than 2,000 years and have clearly declined in the Qing Dynasty. For the current inheritance of the Zheng tunes, there is continuous inheritance and improvement of the Zheng tunes only in Anyang, Henan. The locals call them the "Nanyang drum tunes", and the development of this kind of music also fully reflects the combination of "Boutou tunes" and "Paizi tunes", that is, the collision and fusion between the expression of pure lyrics and the expression of pure music. In the process of teaching Henan Zheng tunes, it is necessary for teachers to explain the historical development process and the influence of folk customs in a detailed and systematic way to help students understand the meaning of the tunes. In addition, students can better understand the Central Plains culture and understand the impact of historical development through the study of traditional Zheng tunes. And this kind of study also helps students to better inherit and innovate Zheng tunes in the future, and has a non-negligible effect on students' systematic master of the Zheng tunes;

Secondly, Zhejiang Zheng tune is also a brand of traditional Zheng tunes that is developing well. From a historical perspective, the Zheng tunes in Zhejiang were inflowed from Henan in the Wei, Jin and Southern and Northern Dynasties, and gradually merged with Zhejiang's local vocal performance in the process of development. Zhejiang Zheng tunes were mainly influenced by the rap art in Zhejiang. The performance of Zheng tunes is called "Tanhuang" by the locals. Zhejiang Zheng tunes are performed by the players through the combination of fast hooks, brackets and wipes of the strings by their thumbs, index fingers and middle fingers, which is also known as the obvious feature of traditional Zhejiang Zheng tunes. The tunes "Jiangjunling" and "Yueergao", which are currently widely circulated and loved, are important representatives of the traditional Zhejiang Zheng tunes. As for the naming of the 
Zhejiang Zheng tunes, due to the prosperous development of local traditional stringed and woodwind instrumental music and the high production of local traditional stringed and woodwind instrumental tunes, the names of the Zheng tunes and the names of the traditional stringed and woodwind instrumental tunes are partially shared. In the process of teaching traditional Zhejiang Zheng tunes, teachers not only need to explain the historical development reasons and the obvious differences between Zhejiang Zheng tunes and the tunes in other regions, but also need to introduce the overall development process of local art to help students cultivate their artistic appreciation ability and guide students to carry out related innovation and integration in the future;

Finally, Hakka culture is an important part of Chinese culture, and it is also a bright pearl in the development of history and culture. The development of Hakka Zheng tunes has an important connection with the change of Hakka culture. Now, Hakka Zheng tunes are also called "Handiao Zheng". From a historical point of view, residents of Hubei and Henan provinces moved to the current Guangdong and Fujian provinces due to the perennial war, becoming the current "Kejia nationality". The impact of the migration is not only the integration of residences, but also the integration of living customs and local arts. Therefore, the development of Hakka Zheng tunes was influenced by the integration of different cultures. In the study of Hakka Zheng tunes, students need to understand the development background to catch the deep feelings of the tunes, try to explore different ways of expression under different emotions and backgrounds, and feel the rhythm, tune and performance method of Zheng tunes. Therefore, in the process of Zheng teaching in colleges and universities, the teaching of traditional Zheng tunes has a nonnegligible effect, which can help students better understand the development and evolution of Zheng tunes, help students understand the influence of different backgrounds on the performing of Zheng tunes, and enable students to improve and learn the performance of Zheng tunes from a technical and artistic perspective.

\section{THE MAIN PROBLEMS IN THE TEACHING OF}

\section{TRADITIONAL ZHENG TUNES IN COLLEGES AND UNIVERSITIES}

On the one hand, there is a lack of teaching of theories about the traditional Zheng tunes. From the perspective of the development of traditional Zheng tunes, there are many genres for traditional Zheng tunes. There are obvious differences in the playing styles, skills and music styles of the different genres. And the genres of Zheng tunes are mainly determined according to their locations. The development and characteristics of different Zheng tune genres are influenced by different regional cultures, folk customs and language systems. Therefore, to study the traditional Zheng tunes, it is needed to firstly understand the development background and evolution process of different genres. Only by fully grasping and understanding the history can we understand the differences between various genres of traditional Zheng tunes and have a deeper understanding of the tunes. However, from the current development situation, it can be seen that in the process of teaching traditional Zheng tunes, most colleges and universities only focus on the teaching of performance skills and methods, and have not conducted teaching of theories and development histories about different genres of Zheng tunes. Many students just know what the genres are, but don't know how they were formed, which is not conducive to the improvement of students' learning effects and students' indepth study of traditional Zheng tunes. As a result, students are hard to accurately play the Zheng tunes of different genres and to show their historical charm.

On the other hand, in the arrangement for Zheng tune lessons, universities usually arrange fewer class hours for traditional Zheng tune teaching, and arrange more class hours for modern Zheng tune teaching. The difference in the curriculum arrangement is obvious, which is not conducive to the students to understand the traditional Zheng tunes more deeply and truly understand the development of the art of Zheng tune playing; and some teachers themselves do not have a deep and accurate understanding of the significance of learning traditional Zheng tunes. They generally consider that the traditional Zheng tunes are relatively simple and there are relatively few playing skills. They have not correctly recognized the necessity of learning traditional Zheng tunes and its meaning to the integrity of students' knowledge system, so there are obvious defects in their teaching; in addition, some teachers themselves have not had the comprehensive and sufficient knowledge about traditional Zheng tunes, and their lack of self-quality leads to their inability to fully explain the traditional Zheng tunes to the students, and it is impossible for these teachers to analyze and explore the traditional Zheng tunes from the perspective of historical development and artistic evolution, which is not conducive to students' in-depth study of traditional Zheng tunes. Therefore, in the future development process, it is necessary to strengthen the teaching of traditional Zheng tunes from multiple aspects, to provide students with more comprehensive explanations, and to continuously improve the effectiveness of traditional Zheng tune teaching and the overall quality of the students.

\section{SUGGESTIONS ON THE OPTIMIZATION OF TRADITIONAL ZHENG TUNE TEACHING IN COLLEGES AND UNIVERSITIES}

\section{A. Improving the Proportion of Theoretical Knowledge Classes}

As mentioned above, the study of traditional Zheng tunes is a relatively complex system, which involves various genres, and there are obvious differences between different genres. But the internal laws of their historical development are similar, that is, different genres have direct connections with the culture, folklore, etc. of different regions. In order to enable students to understand more deeply the origin, development and characteristics of traditional Zheng tunes, teachers need to spend more class time in explaining the theoretical knowledge in teaching. Before teaching the performance skills of each genre, it is necessary for teachers to explain the historical development background of the genre, and link its historical development to current performance method, letting students truly understand the reasons for their different ways of playing and expression. Only by profoundly understanding the root causes and connotations can students truly master the performance methods of traditional Zheng tunes and fully 
reflect the charm of traditional Zheng tunes in their performances. All in all, the learning of traditional Zheng tunes is obviously different from the learning of modern Zheng tunes. The historical nature of traditional Zheng tunes requires teachers to explain them from many aspects. The separate explanation of performance skills is not much meaningful for students, which is not conducive to students' inheritance of traditional Zheng tunes and their innovative performances of the Zheng tunes in the future, and not conducive to the diversified development of art.

\section{B. Improving Teachers' Cognition of Traditional Zheng Tunes}

On the one hand, because some teachers' cognition of traditional Zheng tunes is not accurate enough, they will not introduce and explain traditional Zheng tunes in a comprehensive and large-scale manner in their daily teaching process, which has led to many students' lack of systematic cognition of the development, evolving and merging of traditional Zheng tunes. And because the performance skills in traditional Zheng tunes are not as rich as those in modern Zheng tunes, and teachers pay too much attention to the teaching of skills, so there are few explanations for traditional Zheng tunes. Based on this situation, teachers first need to change their attitudes, truly recognize the necessity and importance of traditional Zheng tune learning, and gradually increase the proportion of courses in traditional Zheng tunes in the specific teaching process, and let the students gradually have better understanding of traditional Zheng tunes;

On the other hand, the quality of some teachers themselves is not high, and their knowledge about traditional Zheng tunes is insufficient, so they are unable to teach students accurately. For this situation, it is required that teachers actively study the knowledge about traditional Zheng tunes in their spare time, make their knowledge system more comprehensive, and gradually integrate the knowledge into their teaching process. The schools can also conduct regular training and assessment for teachers, and organize teachers to actively conduct teaching visits and study to learn the mature and excellent teaching experience, which is conducive to the innovation and improvement of teaching, thus continuously improving the comprehensive effectiveness of the teaching of Zheng.

\section{Stimulating Students' Interest in Learning Through Diversified Teaching Methods}

Because the traditional Zheng tunes are little introduced by the teachers, the students are less interested in the study and research of the traditional Zheng tunes. The time and energy they spend in traditional Zheng tune learning is relatively less, and their subjective initiative of learning and practice is low, which affects the learning effect of students. Therefore, in the daily teaching process, teachers should cultivate students' interest in the learning of traditional Zheng tunes, and improve the students' learning effectiveness and their cognition and exploration of traditional Zheng tunes by letting them generate interest and form good habits. For example, in the teaching of Henan traditional Zheng tunes, teachers can conduct simple demonstrations and encourage students to figure out the performance skills and styles of the tunes. Then teachers can guide students to spontaneously conduct researches on this kind of Zheng tunes by referring to relevant books, searching information on the Internet, etc., and give students enough opportunities to show what they have got from their researches in the next class, and guide students to discuss in class, etc., making the classroom teaching more interesting, and helping students discover the charm of traditional Zheng tunes through an active class atmosphere and develop an interest in learning. In addition, teachers can also use the multimedia technologies to attract students' attention in the specific teaching process. For example, in the introduction of Henan Zheng tunes, teachers can use multimedia to show the development history, folk customs and current development of Henan, laying a good foundation for the students to understand the Zheng tunes in Henan. And the multimedia technologies can also make the theoretical knowledge not so boring.

\section{Organizing a Variety of Traditional Zheng Tune Theme Activities}

In the specific teaching process, in order to improve the teaching effectiveness of traditional Zheng tunes in colleges and universities, teachers can also organize various activities at different teaching nodes, thus making students pay more attention to traditional Zheng tunes and have more interest in them. At first, teachers can hold activities only in their own classes, divide the class into different performance groups, and encourage the groups to perform traditional Zheng tunes. In order to make the activities more interesting, teachers can also change the performance mode, for example, students can tell about the development and changes of Zhejiang traditional Zheng tunes by performing stage dramas; and students can play traditional Zheng tunes collectively in traditional costumes. In the specific process, teachers need to guide the students to make full use of their imagination and play traditional Zheng tunes in a variety of forms, making the learning and performance of traditional Zheng tunes more interesting. After the class activities are mature, teachers can also try to organize activities, competitions, etc. in larger scales. Through various activities, the learning effect of students can be effectively enhanced and their understanding and cognition of traditional Zheng tunes can be deepened.

\section{CONCLUSION}

Traditional Zheng tunes play an important role in the teaching of Zheng in colleges and universities. Through this article, I hope to promote the improvement of the effect of traditional Zheng tune teaching, and attract more and more attention and exploration of traditional Zheng tunes.

\section{REFERENCES}

[1] Zeng Yuzhen. On the Importance of Traditional Zheng Tunes in the Teaching of Guzheng in Colleges and Universities [J] Song of the Yellow River, 2017, 20: pp.28-29. (in Chinese)

[2] Bao Xiaoyuan. Preliminary study on the psychological dilemma of guzheng playing and appreciation in the teaching of guzheng in colleges and universities [J] Song of the Yellow River, 2017, 03:pp.24-25. (in Chinese)

[3] Chen Zhanyu. Analysis of the Importance of Music Analysis in Guzheng Teaching [J] Artists of China, 2017, 03: pp.87-88. (in Chinese) 
[4] Rong Shao. Strategies to Promote the Effectiveness of Guzheng Teaching in Colleges and Universities [J] Art Evaluation, 2017, 23: pp.110-111. (in Chinese) 